\title{
A Comparative Study for Some Technical Characteristics for Salto Bwd Str and Double Salto Bwd Str Dismount on Horizontal Bar
}

\author{
Youssef Mohamed Youssef*
}

\begin{abstract}
The Aim Of The Research Was To Carry Out The Mechanical Characteristics Of Salto Bwd Str And Double Salto Bwd Str And Compare Them. The Research Depends On Descriptive Method By Kinetic And Kinematic Analysis . Research Sample Was Selected Intentionally From The Egyptian Gymnastics Team. Performing Horizontal Bar Sample Elements. The Measured Mechanical Variables Was: Angular Change Of Hip Joints - Angular Change Of Shoulder Joints -Angular Velocity-Angular Speed-Kinetic Energy-Inertia. The Research Conclusions Through The Results of The Mechanical Analysis And Comparison Between The Two Sample Elements the researcher Concludes The Following : There Is A Great Convergence In The Values Of The Measured Mechanical Variables. There Are Differences Between The Two Elements In Power Exert Timing Over The Element - Coaches can increase their player's high difficult elements through the use of the values of variables that have been studied.
\end{abstract}

\section{Introduction}

$\mathrm{G}$ ymnastics Is One of The Sports That Has A Remarkable Development In The Diversity of The Movement Skills On Various Apparatus And Proceeding of Various Related Sciences Development In The Physical Education Field And High Level Sport. The Researcher Noticed That Degree of Difficulty In Gymnastic elements Has Increased Dramatically During The Last 30 Years. From This Point And Through The Work of A Researcher In The Field of Gymnastics Researcher Noted A Major Development In Gymnastics Code, Especially On A Horizontal Bar Where Modern International Code Obliges The Gymnast To Perform elements from five groups one of them is dismount group that the player has to perform dismount With At Least Difficulty (D) To Take The Requirement Degree Which Can Significantly Affect A Gymnast's Degree In The Case of Loss The Degree of The Requirement, So all Gymnastics Coaches Found It Difficult To Improve The Performance of The Minimum Degree Requirements To Reach The Required

* Lecturer, Sports Health Sciences Department, Faculty of Physical Education, Minia University, Egypt.
Difficulty. So Researcher Will Analyze Some Dismount Elements On Horizontal Bar, Which Can Evolve To Reach Minor Difficulties And Medium To High And Difficulties That Check The Value of The Gymnast Dismount Requirement . The Researcher Has Chosen The Following Elements:

1. Salto Bwd Str Dismount (Horizontal Bar).

2. Double Salto Bwd Str Dismount (Horizontal Bar).

Therefore, The Researcher Will Analyze The Performance of the two sample elements To Identify the Technicians' Characteristics Then Compared them

To Identify the Points Of Congruence And Difference That Can Benefit Those Involved In The Process of Gymnastics. Training.

\section{Research Objectives}

1. Identify The Mechanical Characteristics of Salto Bwd Str Dismount on Horizontal Bar.

2. Identify The Mechanical Characteristics of Double Salto Bwd Str Dismount on Horizontal Bar.

3. Compare The Mechanical Characteristics of Salto Bwd Str And Double Salto Bwd Str. 


\section{$\underline{\text { Research Methodology }}$}

The Researcher Depends On Descriptive Methods By Kinetic And Kinematic Analysis .

\section{Research Sample}

\section{Gymnast Sample}

Research Sample Was Selected Intentionally From The Egyptian Gymnastics Team . One Gymnast Was Distinct In Performing Horizontal Bar sample Elements. He Was 19 Years Old And Weighed 55 Kilograms With A Height of $177 \mathrm{Cm}$. He Performed The Elements Under Discussion For Five Times Individually . The Best Attempts for analysis Were Selected By Some International Judges .

\section{Elements Sample}

1. Salto Bwd Str Dismount On Horizontal Bar. 2. Double Salto Bwd Str Dismount On Horizontal Bar.

\section{Procedures of Photographing Process}

- The Researcher Used a Video Camera "Sony", Operating With a Frequency of (25
Frames / Sec). The Player Was Filmed Individually For 5 Attempts of The Two Investigated Elements .

- The Lens of The Camera Was Installed Along The Axis of The Horizontal Bar (Along The Transverse Axis of The Player) And In The Same Height And At a Distance That Allows The Two Investigated Elements Within The Field of Photography.

- There Were No Deviations In The Field of Photography By Water Balance Existing At Camera Holder.

- Using Light Reflexive Labels on The Joints.

- System Calibration Was Designed Related To The Investigated Elements.

\section{Biomechanical Variable}

- Angular Change of The Hip Joints (Degree).

- Angular Change of The Shoulder Joints(Degree)

- Angular Velocity (Degree/S) .

- Angular Speed (Degree/S).

- Angles of Release (Degree) .

- Kinetic Energy.

- Inertia (N.M2) . (J)

Table (1)

Biomechanical sample variables Equation

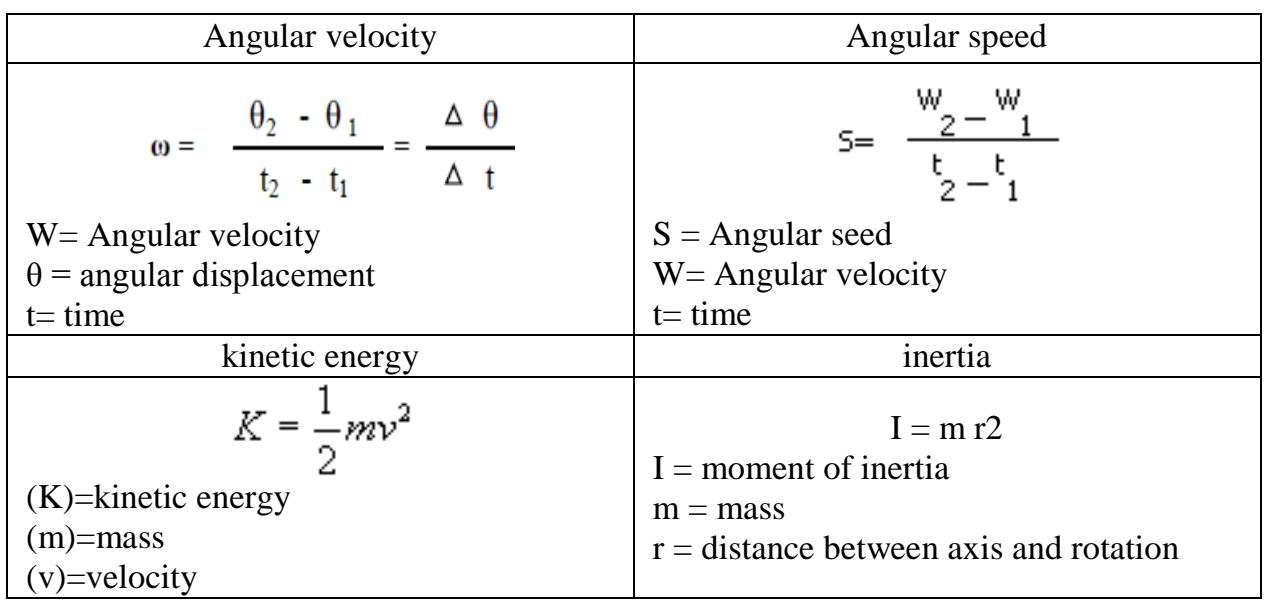




\section{Salto bwd str Dismount Analysis Results}

Figure (1)

Stick Figure for Salto Bwd Str Dismount

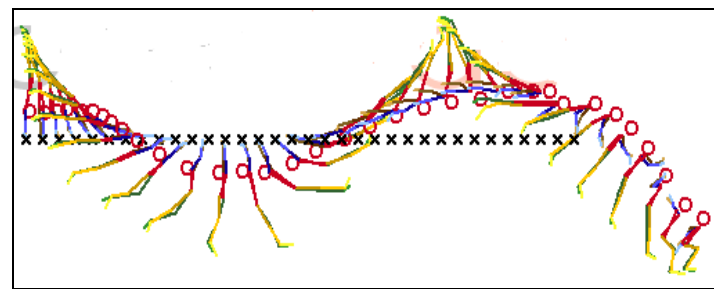

Figure (2)

Angles Of Release position During Salto Bwd Str

Dismount

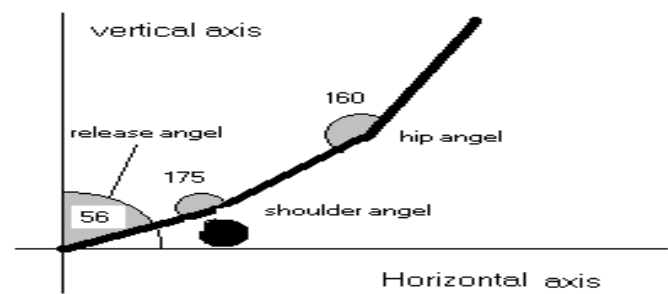

1. Critical points During Salto Bwd Str Dismount

\begin{tabular}{|c|c|}
\hline Critical points & Degree \\
\hline Release Angle During Release Moment & 56 \\
\hline Hip Joints Angel During Release Moment & 160 \\
\hline Shoulder Joints Angel During Release Moment & 175 \\
\hline
\end{tabular}

\section{Hip And Shoulder Joints Angel Change During Salto Bwd Str Dismount}

Graph (1)

Hip Joint Angel Change During Salto Bwd Str Dismount (degree)

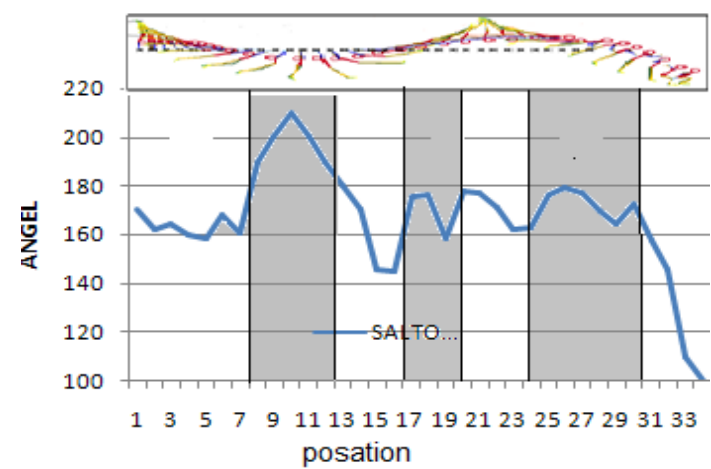

Through Graph (1) The Angular Change of The Hip Joints Appeared That The Gymnast Started The Performance With 160-180 Hip Joints Degree Till Position Number (7) Then The Gymnast Started To Extend The Hip Up To 220 Angle Degree In Order To Perform The Back Movement For Recovery Phase Of The Hip And Carried Out By The Gymnast To Collect The Necessary Power For The Element .Then Begins In Hip Flexion From (220 To 140 Hip Joints Degree) In 0.4 Seconds Then The Gymnast Release From The Horizontal Bar And Keep His Hip Angle From (160 To 180 Degree) In Order To Preserve His Body Straight.
Graph 2

Shoulder Joints Angel Change During Salto Bwd Str Dismount (degree)

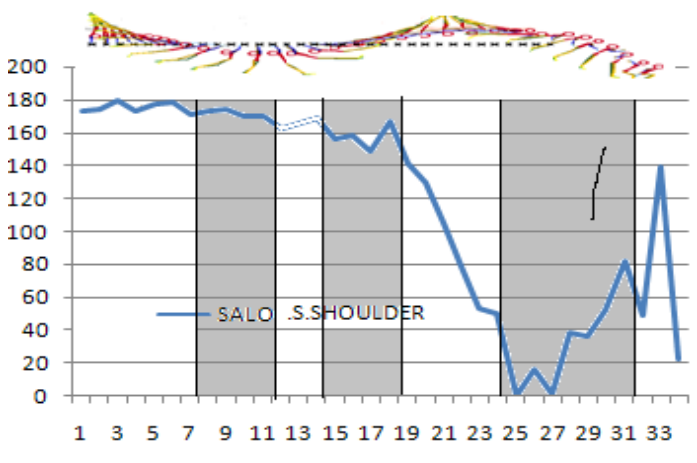

Through Graph (2)the angular change of the shoulder joint appeared that the gymnast started the performance with (160-180) shoulder degree till position number (15) Then the gymnast release from the horizontal bar and started to flex the shoulder from (150 degree ) up to (10 angle degree)in a fast way in order to perform the shoulder flexion which helps the gymnast to have high speed rotation Then the gymnast begins to extend the shoulder to stop rotation and have a good landing . 


\section{Angular Velocity and Angular Speed For Salto Bwd Str Dismount}

Graph (3)

Angular Velocity during Salto Bwd Str Dismount (degree/s)

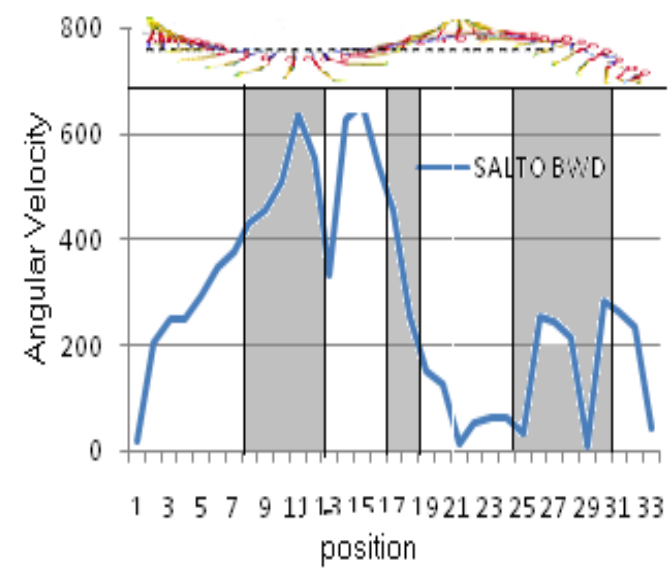

Through Graph (3) The Values Of Angular Velocity Increases During The Introductory Part Of The Element And Then The Gymnast Extended His Hip Up To 220 Hip Angle Degrees, Thus Reducing Angular Velocity. Then The Values Of Angular Velocity Increases Because the Gymnast Flex the Hip, to gain The Necessary Power required for the Element

Through Graph (4) Is Clear To Us The Presence Of Volatile Increases In Angular Speed Since
Graph (4)

Angular Speed during Salto Bwd Str Dismount (degree/s)

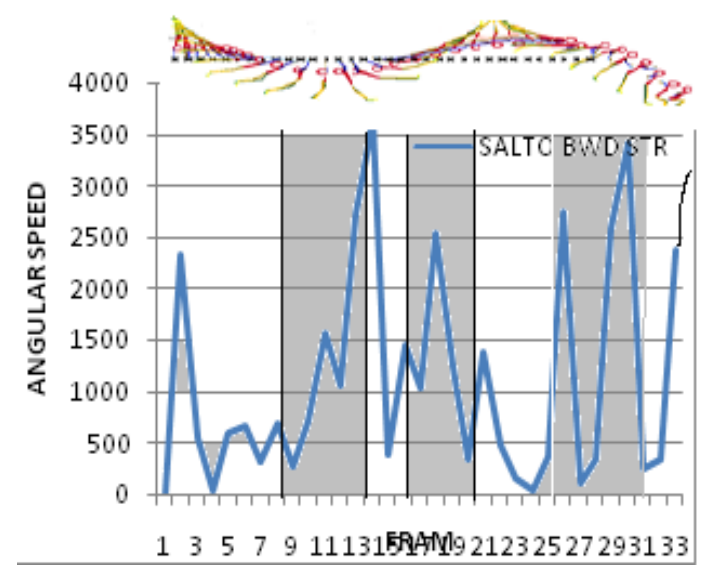

The Beginning Of The Element And Then Recorded Greatest Angular Velocity Value Which Makes Sense Because The Gymnast Moves In The Gravity Direction, Which Increases The Angular Speed. Then Angular Speed Begins To Decline When The Gymnast Body Rising Against Gravity, Until The Moment Of Release. Then The Angular Speed Value Increase Again After The Gymnast Arrived To The Maximum Height .

\section{Kinetic Energy And Inertia For Salto Bwd Str Dismount}

Graph (5) Kinetic Energy values During Salto Bwd Str Dismount (J)

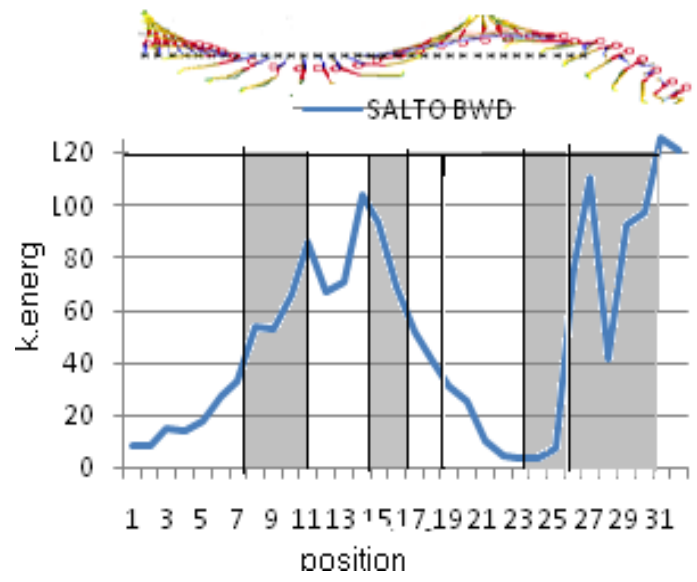

Through Graph (5) The Kinetic Energy Values Increase From The Beginning Till Farm Number (15) Which Is Located Under The Horizontal Bar Because The Kinetic Energy Increases Significantly When Gymnast's Body
Graph (6)

Inertia values During Salto Bwd Str Dismount (N.M2)

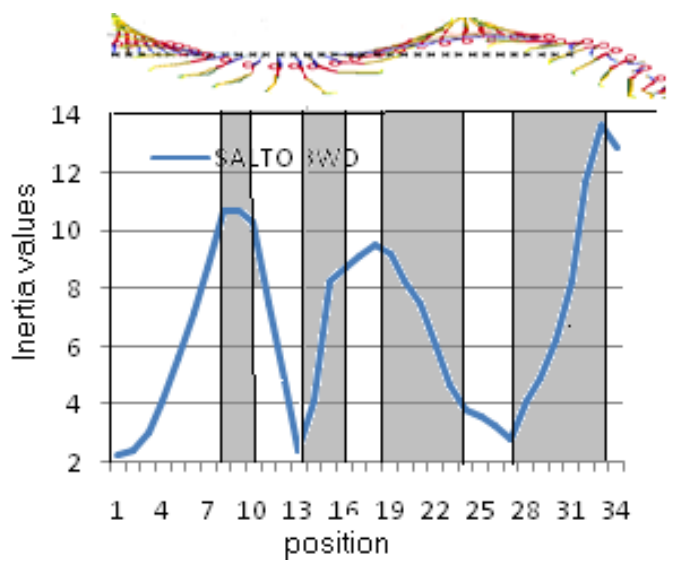

Moves In The Direction Of Gravity And Then Followed By A Decrease In The Values Because The Gymnast's Body Moves Against Gravity And Thus Kinetic Energy Values Decreased Till Position Number (25) To Zero, 
Which Occur During The Maximum Height For The Gymnast's Body During Flight Then Follows By Increase In The Values of The Kinetic Energy Because The Gymnast Body Moves In The Direction of Gravity.

Through Graph (6) The Inertia Values Begin To Increase From The Beginning To The Position Number (10) Because The Gymnast Increase The Radius of Rotation To Increase The Force Which Used By The Gymnast To Perform The
Element And Therefore The Values of Inertia Has Been Increased .Followed By A Decrease In The Values of Inertia Because It Seemed The Movement of The Gymnast Is Against Gravity And The Gymnast Shorten The Radius To Overcome Gravity And Thus There Is A Decline In The Values of Inertia Then Follows By Another Increase Because The Gymnast Extend His Hip In Order To Preserve His Body Straight .

\section{Double Salto Bwd Str Dismount Analysis Results}

Figure (3)

Stick Figure for Salto Double Bwd Str Dismount

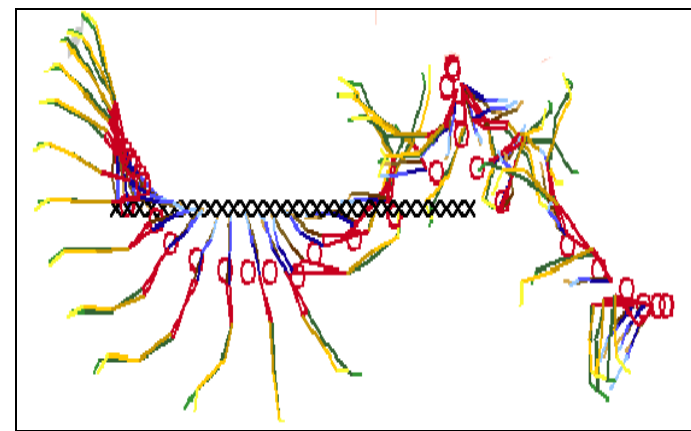

Figure (4)

Angles Of Release position During double Salto Bwd Str Dismount

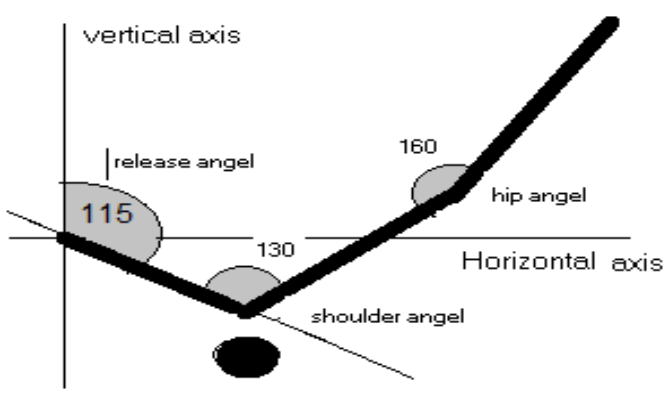

1. Critical Points During Double Salto Bwd Str Dismount

\begin{tabular}{|c|c|}
\hline Critical points & Degree \\
\hline Release Angle DURING Release Moment & 115 \\
\hline Hip Joints Angel DURING Release Moment & 160 degree \\
\hline Shoulder Joints Angel During Release Moment & 130 degree \\
\hline
\end{tabular}

\section{Hip and Shoulder Joints Angel Change During Double Salto Bwd Str Dismount}

Graph ( 7)

Hip Angel Joints Change During Double Salto Bwd Str Dismount (degree)

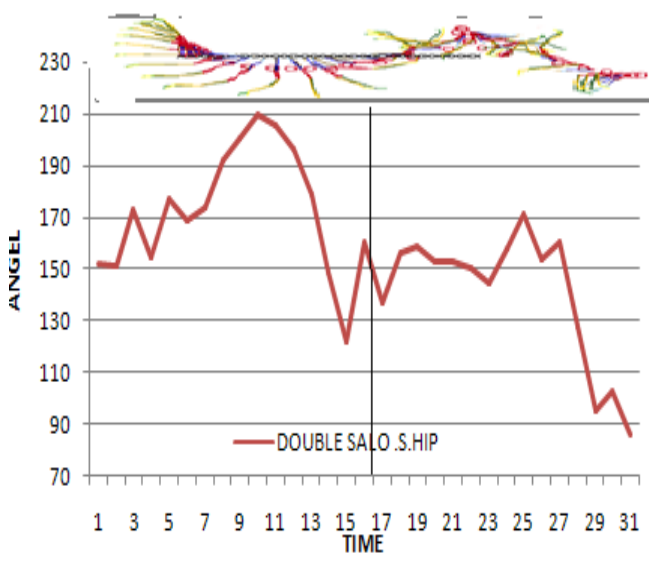

Through Graph (7)The Angular Change of The Hip Joints Appeared That The Gymnast Started The Performance With(160-180 Degree) Till Position Number (6) Then The Gymnast Started
Graph (8)

Shoulder Angel Joints Change During Double Salto Bwd Str Dismount (degree)

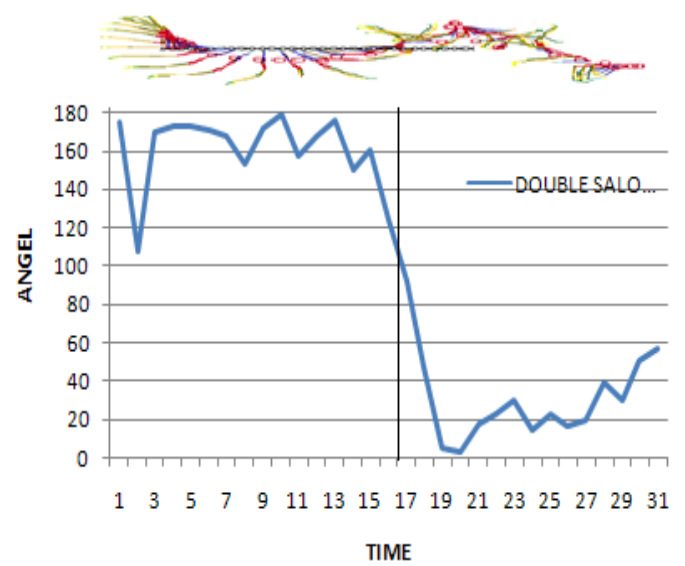

To Extend The Hip Up To Angle ( 210 Degree) In Order To Perform The Back Movement For Recovery Phase Of The Hip And Carried Out By The Gymnast To Collect The Necessary 
Power For Performing The Element Then Begins In Hip Flexion From ( 220 To 120 Degree)In0.4seconds Then The Gymnast Release From The Horizontal Bar And The Gymnast Keep The Hip Angle From (140 To 180 Degree) In Order To Preserve His Body Straight

Through Graph (8)The Angular Change of The Shoulder Joints Appeared That The Gymnast Started The Performance With (150-180
Shoulder Degree ) Till Position Number (15) Then The Gymnast Release From The Horizontal Bar And Started To Flex The Shoulder From (150 Degree ) Up To (5 Angle Degree )In A Fast Way In Order To Perform The Shoulder Flexion Which Helps The Gymnast To Have High Speed Rotation Then The Gymnast Begins To Extend The Shoulder To Stop Rotation And Have A Good Landing .

\section{Angular Velocity and Angular Speed for Double Salto Bwd Str Dismount}

\section{Graph (9) \\ Angular Velocity For Double Salto Bwd Str Dismount (degree/s)}

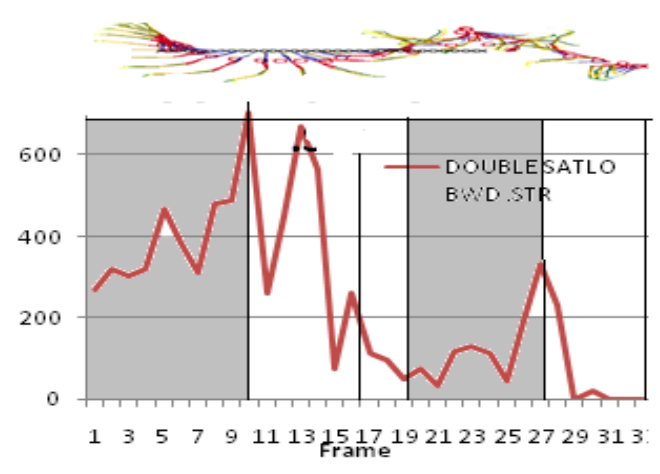

Through Graph (9) Is Clear That Values Of Angular Velocity Increases During The Introductory Part Of The Element And Then The Gymnast Extended The Hip To (220 Degree) Thus Reducing Angular Velocity Then The Values of Angular Velocity Increases Because The Gymnast Flex The Hip, To Collect The Necessary Power For The Element Then The Gymnast Release From The Horizontal Bar And The Gymnast Keep The Hip Angle From (140 To 180 Degree) In Order To Preserve His Body Straight .

Through Graph (10) Is Clear To Us The That Values of Angular Speed Increased From The
Graph (10)

Angular Speed For Double Salto Bwd Str Dismount (degree/s)

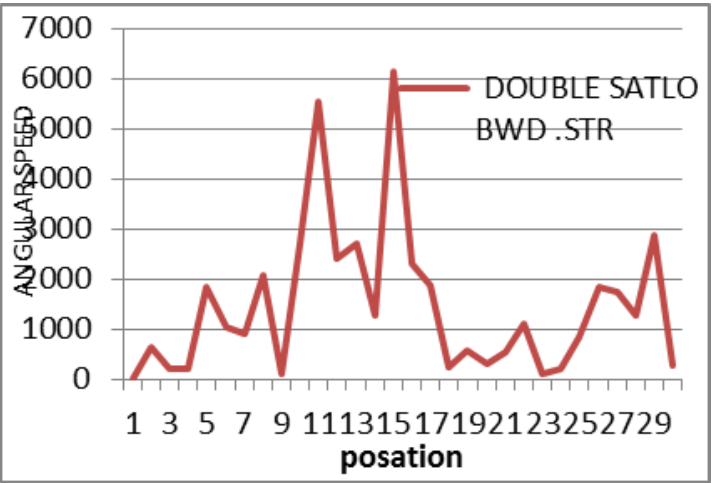

Beginning of The Element Till Position Number (8) And Then Angular Speed Begins To Decline When The Gymnast Body Rising Against Gravity Position Number (12)And Then Increased Because Of The Recovery Phase (Hip Flexion)

And Then Recorded Greatest Angular Velocity Value Which Makes Sense Because The Gymnast Moves In The Gravity Direction, Which Increases The Angular Speed .And Also Because of Hip And Shoulder Flexion. Then The Angular Speed Value Decrease After The Gymnast Finish The Two Saltos And Preparing For The Landing 


\section{Kinetic Energy and Inertia for Double Salto Bwd Str Dismount}

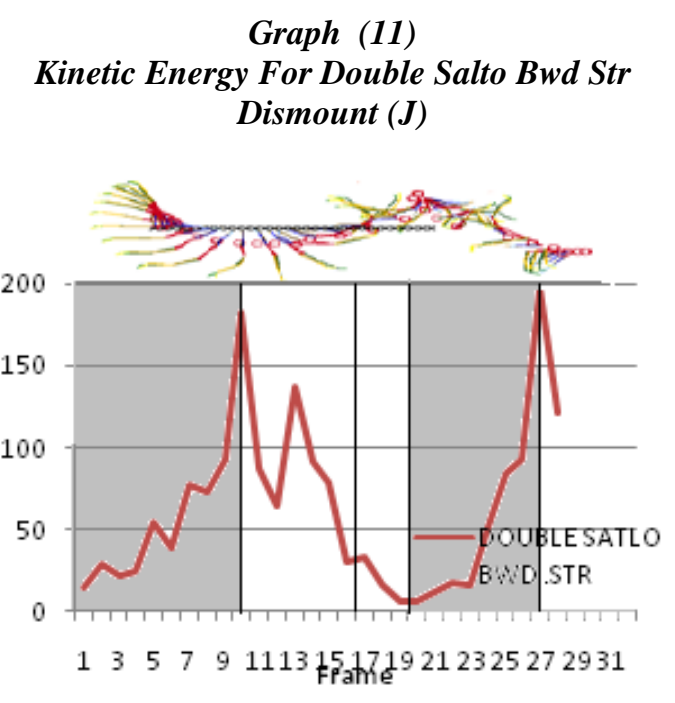

Through Graph (11)Values of The Kinetic Energy Increase From The Beginning Till Farm Number (10) Which Is Located Under The Horizontal Bar Because The Kinetic Energy Increases Significantly When Rotation of The Gymnast's Body Moves In The Direction Of Gravity And Then Followed By A Decrease In The Values Because The Gymnast's Body Moves Against Gravity And Thus Kinetic Energy Values Decreased Till Position Number (25) The Kinetic Energy Value Decreased To Zero, Which Occur During The Maximum Height For The Gymnast's Body During Flight Then Follows By Increase In The Values of The Kinetic Energy Because The Gymnast Body Moves In The Direction of Gravity.

\section{Compare the Two Sample Eelements}

\section{Compare the Critical Points}

Figure (5) Angles of Release position During Salto Bwd Str and Double Salto Bwd Str

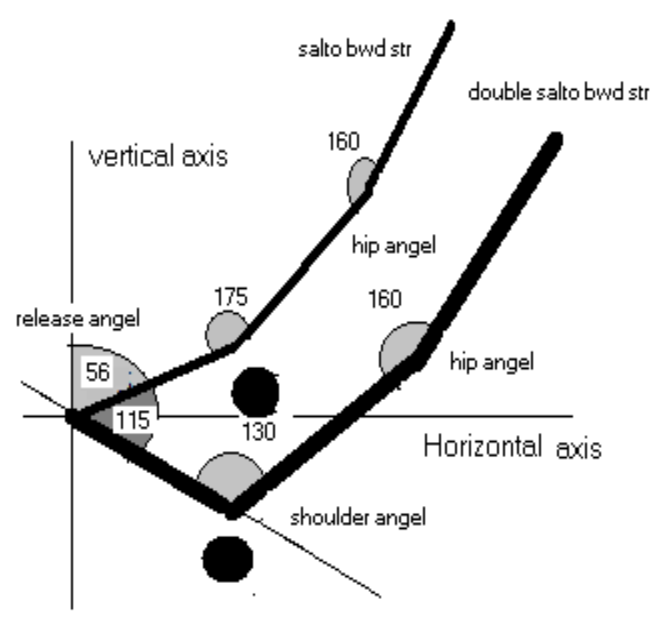

Graph (12)

Inertia For Double Salto Bwd Str Dismount (N.M2)

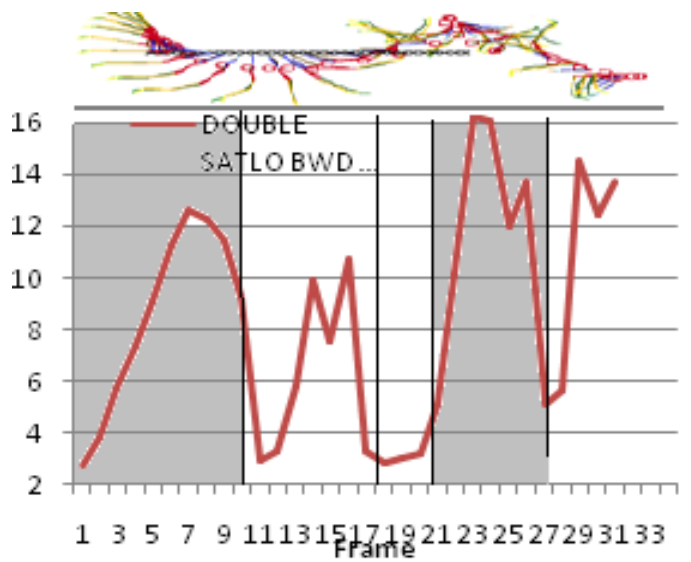

Through Graph (12), The Inertia Values Begin To Increase From The Beginning To The Position Number (10) Because The Gymnast Increase The Radius of Rotation To Increase The Force Which Used By The Gymnast To Perform The Element And Therefore The Values of Inertia Has Been Increased. Followed By A Decrease In The Values of Inertia Because The Movement of The Gymnast Was Against Gravity And The Gymnast Shorten The Turning Radius To Overcome Gravity And Thus There Is A Decline In The Values of Inertia .Then Follows By Another Increase Because The Gymnast Extend The Hip In Order To Preserve His Body Straight.
Table (2)

Critical Points For Release Position During Salto Bwd Str And Double Salto Bwd Str

\begin{tabular}{|c|c|c|}
\hline $\begin{array}{c}\text { CRITICAL } \\
\text { POINTS }\end{array}$ & $\begin{array}{c}\text { Double } \\
\text { salto bwd }\end{array}$ & Salto bwd \\
\hline $\begin{array}{c}\text { Release Angle } \\
\text { (degree) }\end{array}$ & 56 & 115 \\
\hline $\begin{array}{c}\text { Shoulder Joints } \\
\text { Angel During } \\
\text { Release } \\
\text { Moment(degree) }\end{array}$ & 175 & 130 \\
\hline $\begin{array}{c}\text { Hip Joints Angel } \\
\text { During Release } \\
\text { Moment(degree) }\end{array}$ & 160 & 160 \\
\hline
\end{tabular}


Through a table (2) and fig (5) we note the following:

1. There is a significant difference in the release angle between two elements reaching (56 degrees) during Salto Bwd Str and (115 degrees) during the double Salto Bwd Str because the player needs high speed rotation to help in completing the double salto unlike Salto Bwd Str that does not need the high speed rotation .

2. for the same reason , and the same Explanation we find that there is a large difference in the angle of the shoulder joint during a moment of releasing between the two elements and also angle of the shoulder joint during the double Salto Bwd Str was bigger than angle of the shoulder joint during the Salto Bwd Str .

3. there is no different in the hip joint angle during the moment of releasing because the player needs to keep his body straight during the two elements

\section{Compare the Hip and Shoulder Joints Angel Change}

Graph (13)

The Hip Joints Angel Change During Salto Bwd Str And Double salto Bwd Str (degree)

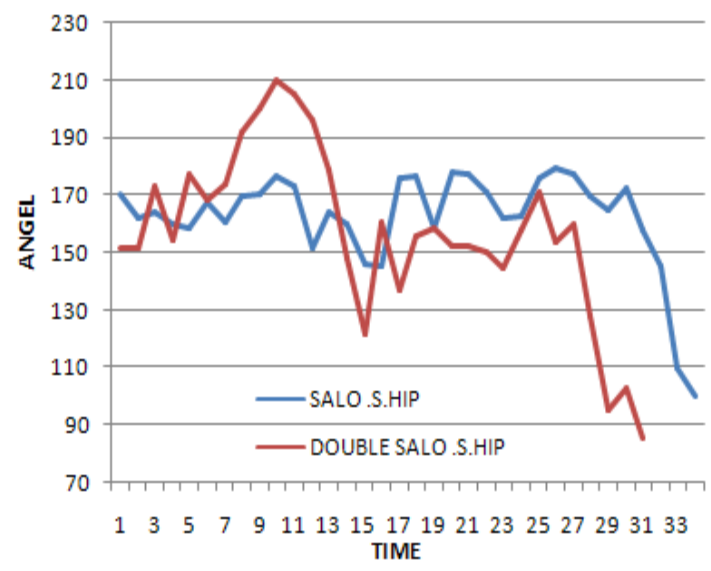

Through Graph (13)Clear That There Is A Near Consensus Among Angular Change of The Hip Joints For The Two Elements But The Difference Is In The Hip Flexion As Follows: Hip Flexion Started During Position Number (10) In Salto Bwd Str From( 205 Degree) To (145 Degree) During (0.4) Seconds While Started In Double Salto Bwd During Position Number (10) From (210degree) To (120 Degrees) Directly Through ( 0.4 Seconds) That Means Hip Flexion During The Two Elements Begin In The Same Time, But With The Increase In The Value of Angles of The Hip In Double Salto Bwd.

Through Graph (14) Its Clear That There Is A Near Consensus Among Angular Change of The Shoulder Joints For The Two Elements But The Difference Is In The Shoulder Flexion As Follows:
Graph (14)

Shoulder Joints Angel Change During Salto Bwd Str And Double salto Bwd (degree)

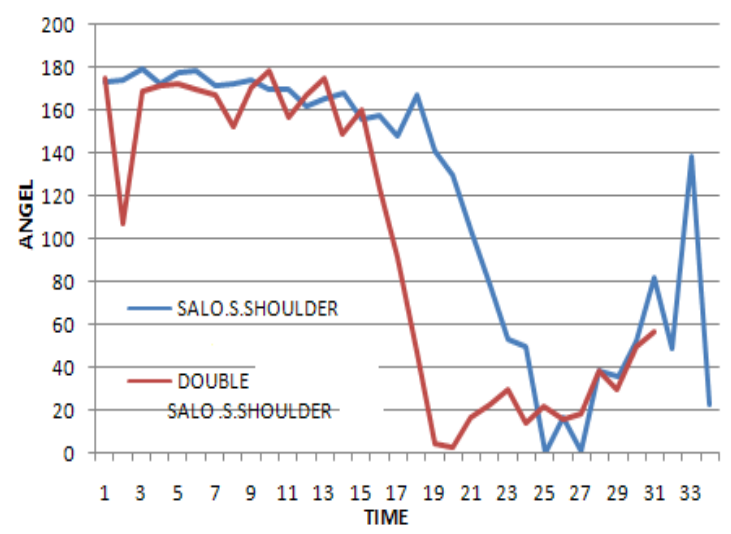

- Shoulder Flexion Started During Position Number (19) In Salto Bwd Str From ( 170 Degree) To (50 Degree) And Then (5 Degree) During A Time of 0.48 Seconds .

- Shoulder Flexion Started During Position Number (16) In Double Salto Bwd Str From ( 170 Degree) To (4degree) During A Time of 0.32 Seconds .

This Means That The Shoulder Flexion For Double Salto Bwd Str Happened Little Bit Early And In A Short Time And At Larger Angles,

Which Means The Double Salto Bwd Str Need A Larger Force And Stronger Shoulder Muscles To Perform Double Salto Bwd Str After Salto Bwd Str 


\section{Compare Angular Velocity Angular Speed Values}

Graph (15)

Angular Velocity Values For Salto Bwd Str And Double Salto Bwd Str(degree/s)

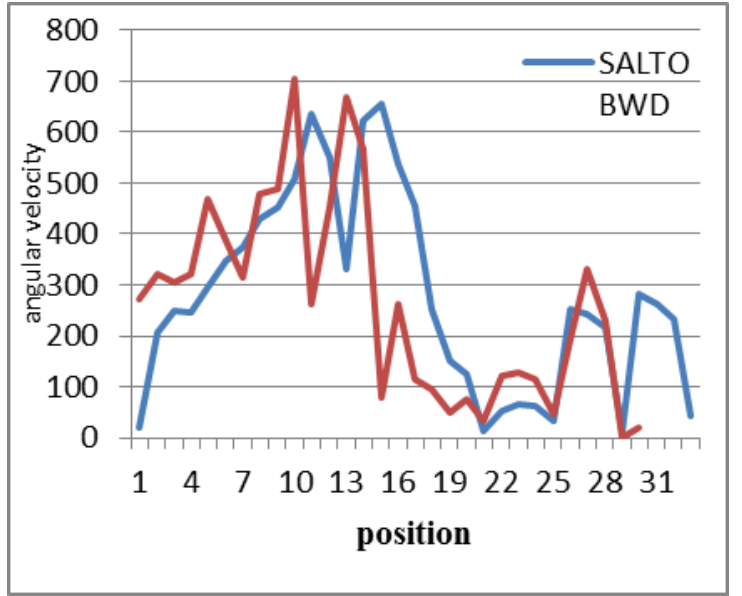

Through Graph (15) That Clear To Us That There Is A Near Consensus Among Angular Velocity Values In The Timing of The Increase And Decrease of The Two Elements But The Difference Is In The Following

1. There Is A Clear Increase of The Angular Velocity of Double Salto Bwd Str Since The Beginning of The Performance To Position Number(15)

2. The Difference Decreased Between The Values of Angular Velocity For The Two Elements Starting From Position Number (16) To Position Number(25) Then The Difference Between The Two Values Increased Near The End of Two Elements

Through Graph (16) We Find That :There Is A Similarity In The Increase And Decrease Of The Values of The Angular Velocity Of The Two Elements But With Difference In Values Is As Follows:
Graph (16)

Compare Angular Speed Values For Salto Bwd Str And Double Salto Bwd Str (degree/s)

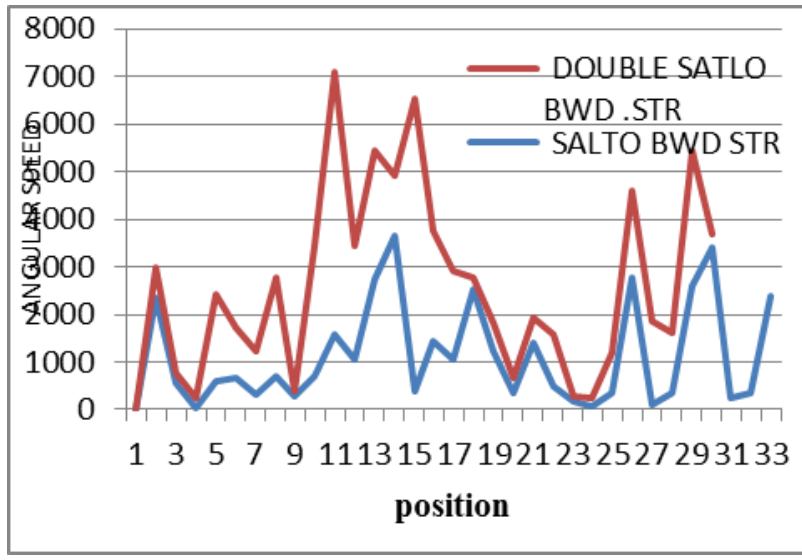

There Is A Similarity In The Increase And Decrease Of The Angular Velocity Values For The Two Elements But With Some Differences In Several Positions In The Benefits of Double Salto Bwd As Follows:

1. Between The Positions Number (4-10)

(Preparation Phase)That The Period During The Movement For The Rehabilitation of The Body To Capture Power And Speed Necessary To Perform The Element Between The Positions Number (10-19)(Hip Flexion Phase)The Player Try To Get The Needed Power And Speed To Perform The Element

2. Between The Positions Number (25-30)( Flight Phase) It Makes Sense That Angular Velocity of Double Salto Bwd Is More Than Salto Bwd 
4. Compare Kinetic Energy and Inertia Values

Graph (17)

Compare Kinetic Energy Values For Salto Bwd Str And Double Salto Bwd Str(J)

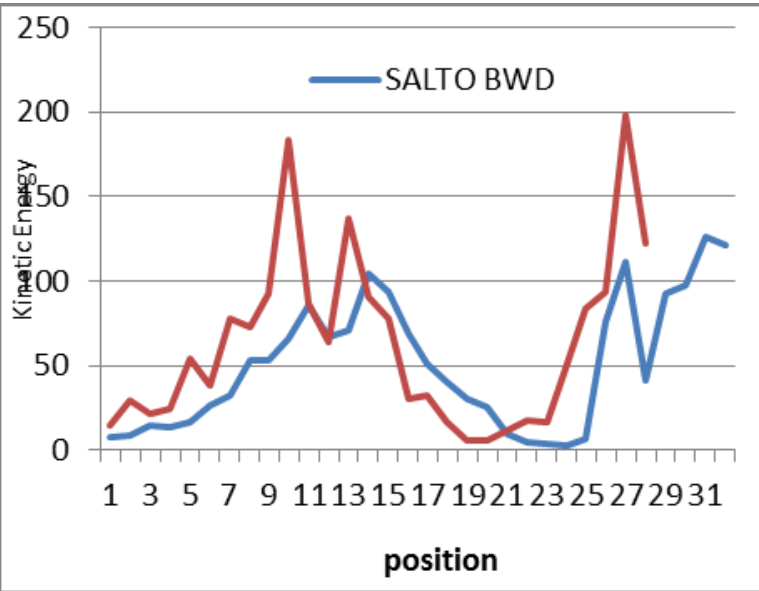

Through Graph (17)We Find That There Is Symmetry In The Increase And Decrease Of The Values of The Kinetic Energy of The Two Elements But With A Light Difference In Values For The Benefit of Double Salto Bwd Str As Follows:

1. Values of Kinetic Energy of The Two Elements Start Increasing From The Beginning Until The Gymnast's Body Arrival To An Existing Horizontal Bar And To Explain That The Kinetic Energy Increases Significantly When Gymnast's Body Moves In The Direction of Gravity, But Values Vary Is Up Through Salto Bwd Str To (112 J)While Reached During Double Salto Bwd Str To185 (J)And That Mean A Large Deferent Between The Two Elements .

2. The Values Of The Kinetic Energy of The Two Elements Decreased Because The Gymnast's Body Moves Against The Gravity Until The Player Releases From The Horizontal Bar And We Note That The Releasing Time In Double Salto Bwd Str Happened Earlier Than Salto Bwd Str .

3. Values Of The Kinetic Energy Continues To Decrease Until It Reaches The Closest Values To Zero. Upon Gymnast's Body's Arrival To The Maximum Height During Flight And Up
Graph (18)

Compare Inertia Values For Salto Bwd Str And Double Salto Bwd Str (N.M2)

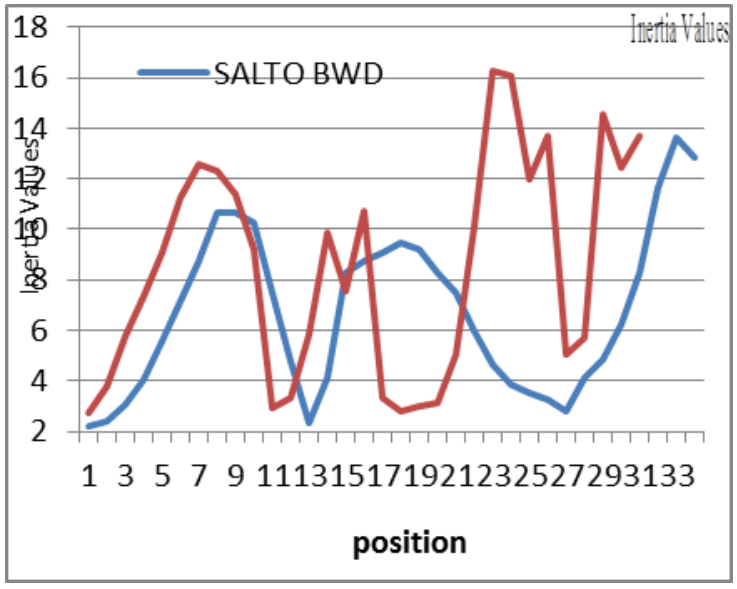

The Value of The Kinetic Energy To Approximately (200 J) While Up Through Salto Bwd Str To (100 J).

Through Graph (18)We Find That: There Is A Similarity In The Values Increase And Decrease of Inertia Of The Two Elements But With A Difference In Values For The Benefit of Double Salto Bwd Str As Follows:

The Differences Between Two Elements Starts From The Moment of Release Where Inertia Values For Salto Bwd Str While Inertia Becoming Increasingly For Double Salto Bwd Str With A Great Extent Because of The Large Difference In Rotational Speed Between The Two Elements .

\section{Compare Elements Parts}

Each elements has been divided into quarters from hand stand to release so that we can compare between the two elements as follows:

Phase one: from hand stand to horizontal axis

Phase two: from horizontal axis to vertical axis under horizontal bar

Phase three: from vertical axis under horizontal bar to horizontal axis

Phase four : from horizontal axis to release 
Table (3)

Critical Points For Release Position During Salto Bwd Str And Double Salto Bwd Str dismount

\begin{tabular}{|c|c|c|c|c|c|c|c|c|}
\hline & \multicolumn{2}{|c|}{ Phase One (AVR) } & \multicolumn{2}{|c|}{$\begin{array}{c}\text { Phase Two } \\
\text { (AVR) }\end{array}$} & \multicolumn{2}{|c|}{$\begin{array}{c}\text { Phase Three } \\
\text { (AVR) }\end{array}$} & \multicolumn{2}{|c|}{$\begin{array}{c}\text { Phase Four } \\
\text { (AVR) }\end{array}$} \\
\hline Element & salto & double & salto & double & salto & double & salto & double \\
\hline Time & $\begin{array}{c}0.36 \\
\text { sec }\end{array}$ & $\begin{array}{c}0.56 \\
\text { sec }\end{array}$ & $\begin{array}{c}0.32 \\
\text { sec }\end{array}$ & $\begin{array}{c}0.32 \\
\text { sec }\end{array}$ & $\begin{array}{c}0.4 \\
\text { sec }\end{array}$ & $\begin{array}{c}0.4 \\
\text { sec }\end{array}$ & $\begin{array}{c}0.24 \\
\text { sec }\end{array}$ & -- \\
\hline $\begin{array}{c}\text { Shoulder } \\
\text { Angel(degree) }\end{array}$ & 174.7 & 161.8 & 170.4 & 165 & 161 & 156 & 153.5 & -- \\
\hline $\begin{array}{c}\text { Hip Angel } \\
\text { (degree) }\end{array}$ & 166.6 & 164.2 & 18.2 & 187 & 170 & 171 & 165 & -- \\
\hline $\begin{array}{c}\text { Angular Velocity } \\
\text { (degree/S) }\end{array}$ & 460 & 341 & 460.2 & 425 & 515.8 & 388 & 414 & -- \\
\hline $\begin{array}{c}\text { Angular Speed } \\
\text { (degree/S) }\end{array}$ & 757 & 688 & 757.8 & 1863 & 1586 & 3173 & 1532 & -- \\
\hline $\begin{array}{c}\text { Kinetic energy } \\
\text { (N.M2) }\end{array}$ & 21.5 & 36 & 53.9 & 83 & 72.7 & 81 & 60 & -- \\
\hline Inertia (J) & 5.4 & 7.1 & 8.2 & 9.6 & 6.2 & 7.1 & 7.9 & -- \\
\hline
\end{tabular}

Through table (3) we note the following:

1. Salto Bwd Str divided into 4 stages from handstand to release from the horizontal bar. the longest phase time is the third phase $(0.4 \mathrm{sec})$ and shortest phase time is the fourth stage $(0.24$ sec)

2. Double Salto Bwd Str divided into 3 stages from handstand to release from the horizontal bar. the longest phase time is the first phase $(0.56 \mathrm{sec})$ and there is no fourth stage because the player releases from the horizontal bar before entering the fourth phase .

3. shoulder angles Mean for all stages of Double Salto Bwd Str element was significantly less than Salto Bwd Str

4. There is a great convergence in the values of the hip angles average for all stages of the two sample elements.

5. The Angular Velocity Mean For Double Salto Bwd Str Stages Were Significantly Lower Than Salto Bwd Str

6. The Angular speed Mean For Double Salto Bwd Str Stages Were Significantly Lower Than Salto Bwd Str element Stages .

7. averages of the kinetic energy and inertia for all stages of Double Salto Bwd Str element was significantly less than Double Salto Bwd Str element .

\section{Conclusions}

Through The Results of The Mechanical Analysis And Comparison Between The Two Sample Elements The Researcher Conclude The Following :
1. There Is A Great Convergence In The Values of The Measured Mechanical Variables .

2. There is a Little Differences Between The Two Elements In Power Exert Timing Over The Element.

3. Coaches can increase their player's high difficult elements through the use of the values of variables that have been studied.

\section{References}

1. Bartlett,Roger : Introduction To Sports Biomechanics: Analysing Human Movement Patterns. (4th Ed). Routledge Taylor And Francis Publishing Group. London And New York. . (2009).

2. Code of Points: International Gymnastics Federation - Switzerland .2008

3. Hamilton, N. And K. Luttgens : Kinesiology Scientific Basic Of Human Motion, Mc GrowHill Companies, New York, 10th Ed .Pp: 3103152002

4. Jun Tsuchiya : Kinetic Analysis Of Back Word Giant Swing On Parallel Bars. Faculty Of Sport Science. Wesda University .2004.

5. Knudson, D. V. And C. Morrison : Qualitative Analysis Human Movement. Human Kinetics, U.S.A., Pp: , 122-125 1997

6. Kolar, E., Andlovic Kolar, K., Štuhec, S : Comparative Analysis Of Selected Biomechanical characteristics Between A Support Backward Swing And Support Swing For The 1 - 1/4 Straddle-Piked Forward Salto 
On The Parallel Bars. Sports Biomechanics, 1 (1): 69 - 78(2002).

7. Spiros Prassas : Giant Swing On Parallel Bars. California State Unv. East Bay Hayward.Usa. 2004.

8. Takei Y, Dunn JH : A 'Kickout' Double Salto Backward Tucked Dismount From The Horizontal Bar Performed By Elite Gymnasts. J Sports Sci. 1997 Aug;15(4):411-25.

9. Timothy Exell, Gareth Irwin, David Kerwin : Force Comparison Of Backward Longswings
On High Bar And Uneven Parallel Bar. Xxv Isbs Symposium 2007, Ouro Preto - Brazil

10. V. M. Zalsiorsky : Kinematics of Human Motion, Human Kinetics, U.S.A., Pp73, 81, 94 1998

11. Ying Liu, Xin Ma, Qi Yan and Ge Wang : Biomechanical Analysis Of Back Handspring To Backward Salto With Triple Twist In Gymnastic Floor Exercise Beijing Research Institute Of Sports Science, People's Republic of China 